\title{
Healing of localized gingival recessions treated with coronally advanced flap alone or combined with either a resorbable collagen matrix or subepithelial connective tissue graft. A preclinical study
}

\author{
Anton Sculean • Ilja Mihatovic • Yoshinori Shirakata • \\ Dieter D. Bosshardt • Frank Schwarz • Gerhard Iglhaut
}

Received: 11 May 2014 / Accepted: 22 July 2014 / Published online: 2 August 2014

(C) Springer-Verlag Berlin Heidelberg 2014

\begin{abstract}
Objectives To histologically evaluate the effectiveness of a porcine derived collagen matrix $(\mathrm{CM})$ and a subepithelial connective tissue graft (CTG) for coverage of localized gingival recessions.

Materials and methods Chronic single Miller Class I-like recessions were created at the buccal at the canines and at the third and fourth premolars in the upper and lower jaws of six beagle dogs. The defects were randomly treated with (1) coronally advanced flap surgery $(\mathrm{CAF})+\mathrm{CM},(2) \mathrm{CAF}+\mathrm{CTG}$, or (3) CAF alone. At 12 weeks, histometric measurements were made, e.g., between a reference point $(\mathrm{N})$ - and the gingival margin (GM) - and the outer contour of the adjacent soft tissue (gingival thickness [GT]).

Results The postoperative healing was uneventful in all animals. No complications such as allergic reactions, abscesses or infections were noted throughout the entire study period.
\end{abstract}

A. Sculean and I. Mihatovic contributed equally to this study and are considered joint first authors.

\footnotetext{
A. Sculean $(\bowtie) \cdot$ Y. Shirakata $\cdot$ D. D. Bosshardt

Department of Periodontology, Dental School University of Bern, Freiburgstrasse 7, 3010 Bern, Switzerland

e-mail: anton.sculean@zmk.unibe.ch

I. Mihatovic $\cdot$ F. Schwarz

Department of Oral Surgery, Heinrich Heine University, Düsseldorf, Germany

D. D. Bosshardt

Department of Oral Surgery and Stomatology, University of Bern,

Bern, Switzerland

G. Iglhaut

Private Practice, Memmingen, Germany

G. Iglhaut

Department of Oral and Maxillofacial Surgery, George Augusta

University, Göttingen, Germany
}

All three treatments resulted in coverage of localized gingival recessions. The histological analysis failed to identify any residues of CM or CTG. The histometric measurements revealed comparable outcomes for N-GM and GT values for all three groups $(\mathrm{CAF}+\mathrm{CM}: 1.04 \pm 0.69 \mathrm{~mm} / 0.68 \pm 0.33 \mathrm{~mm}$; CAF+CTG: $1.15 \pm 1.12 \mathrm{~mm} / 0.76 \pm 0.37 \mathrm{~mm}$; CAF: $1.43 \pm$ $0.45 \mathrm{~mm} / 0.79 \pm 0.24 \mathrm{~mm}$ ).

Conclusions In the used defect model, the application of CTG or $\mathrm{CM}$ in conjunction with $\mathrm{CAF}$ did not have an advantage over the use of CAF alone.

Clinical relevance The use of CAF alone is a valuable option for the treatment localized Miller Class I recessions.

Keywords Soft tissue recession - Collagen matrix · Animal study · Coronally advanced flap · Subgingival connective tissue graft · Histology

\section{Introduction}

Localized gingival recessions are frequently observed in both young and older individuals and their treatment is mainly indicated to improve local plaque control, aesthetic demands or dentin hypersensitivity [1]. Recent systematic reviews have indicated that the coronally advanced flap (CAF) is a predictable technique for obtaining complete root coverage (CRC). Moreover, the combination of CAF with subepithelial connective tissue graft (CTG) or enamel matrix derivative has been shown to result in higher $\mathrm{CRC}$ and improved long-term stability compared with CAF alone [2]. However, the surgical procedure to harvest CTG may be associated with intra- or postoperative complications such as bleeding or paraesthesia at the donor site while the second surgical site needed for CTG harvesting is associated with and increase in patient morbidity and surgical time [3, 4]. 
In order to overcome these shortcomings, a porcine-derived, native collagen matrix $(\mathrm{CM})$ has been introduced. The specific porous network of $\mathrm{CM}$ has been shown to support connective tissue ingrowth (e.g., collagen type-3 fibres) thus resulting in an increase of soft tissue thickness of about $21 \%$ at 60 days [5]. Clinical studies have demonstrated that treatment of single or multiple gingival recessions by means of CAF or modified coronally advanced tunnel (MCAT) in combination with CM may lead to an increase in soft tissue thickness and width of keratinized tissue [6-8]. Moreover, in the treatment of single recessions, the precentage of root coverage obtained with $\mathrm{CAF}+\mathrm{CM}$ were comparable to that obtained with $\mathrm{CAF}+\mathrm{CTG}[6,9,10]$. Furthermore, the results of a preclinical study have indicated that treatment of experimentally created recession defects by means of $\mathrm{CAF}+\mathrm{CM}$ resulted in shorter junctional epithelium and larger amounts of newly formed cementum compared with CAF alone [11]. To the best of nour knowledge, at the time being, there are no histological data comparing the healing of single recessions treated by means of CAF alone or combined with either $\mathrm{CM}$ or CTG.

Therefore, the aim of the present study was to histologically compare soft tissue healing and root coverage following $\mathrm{CAF}, \mathrm{CAF}+\mathrm{CTG}$, and $\mathrm{CAF}+\mathrm{CM}$ procedures for the treatment of localized Miller Class I-gingival recessions in a canine model.

\section{Material and methods}

Animals

For this study, six male beagle dogs (age 12-24 months, mean weight $42 \pm 4 \mathrm{~kg}$ ) with a fully erupted permanent dentition were used. During the entire study period, all dogs were fed once per day with soft-food diet and water ad libitum. The study protocol was reviewed and approved by the appropriate local authorities (Nr. LANUV 87-51.04.2010.A086). The experimental segment of the study started after an adaptation period of 4 weeks.

\section{Study design and experimental phases}

In each animal, one quadrant each of the upper and lower jaws was randomly selected for the surgical creation of single Miller Class I-like gingival recessions at the canine as well as third and fourth premolars (C, P3, P4). After a chronification period of 8 weeks, the resulting defects were randomly allocated to either (1) $\mathrm{CAF}+\mathrm{CM},(2) \mathrm{CAF}+\mathrm{CTG}$, or (3) CAF alone.

The randomization procedure accounted for anterior and posterior sites in both upper and lower jaws and was based on a computer generated list (RandList ${ }^{\circledR}$, DatInf $\mathrm{GmbH}$,
Tübingen, Germany). The animals were euthanized after 12 weeks of healing.

Surgical procedures

Prior to each surgical intervention, intramuscular sedation was accomplished with $0.01 \mathrm{mg} / \mathrm{kg}$ acepromazine (Vetranquil $1 \%$; Ceva Tiergesundheit, Düsseldorf, Germany). Subsequently, anesthesia was initiated according to a standard protocol employing $21.5 \mathrm{mg} / \mathrm{kg}$ thiopental-sodium (Trapanal $2.5 \%$; Altana $\mathrm{GmbH}$, Konstanz, Germany) and inhalation anaesthesia performed by the use of oxygen and nitrous oxide and isoflurane. While anesthetized, all animals received a constant rate infusion of lactated Ringer's solution to maintain hydration. Intraoperative analgesia was performed by intravenous injection of $0.1 \mathrm{mg} / \mathrm{kg}$ piritramid (Dipidolor ${ }^{\circledR}$; JanssenCilag GmbH, Neuss, Germany) and $4.5 \mathrm{mg} / \mathrm{kg}$ carprofene (Rimadyl ${ }^{\circledR}$; Pfizer Pharma GmbH, Karlsruhe, Germany). For postoperative treatment, piritramid (days 1-3) and carprofen (days 4-7) were applied subcutaneously in the same dose as described previously.

Phase $1-$ creation of gingival recessions

At each tooth site, a bone sounding procedure employing a conventional periodontal probe (PCP 12; Hu-Friedy, Chicago, IL, USA) was conducted to clinically estimate the biological width. Following intrasulcular incisions (width: $3 \mathrm{~mm}$ ) at the buccal aspect, the supracrestal gingiva was excised employing a semicircular incision not exceeding the mucogingival junction. Tissue height was about $2 \mathrm{~mm}$, but considered the biological width estimated at each site. Subsequently, all experimental sites were left to heal for 8 weeks. At 7 days after surgery, tooth cleaning was initiated and maintained by the use of a toothbrush three times per week.

Phase 2 - coverage of chronic gingival recession-type defects

After a supragingival/-mucosal cleaning of remaining teeth in both upper and lower jaws using rubber cups and a polishing paste (Zircate ${ }^{\circledR}$ Prophy Paste; Dentsply, Konstanz, Germany), clinical defect height $(\mathrm{CDH})$ was estimated at the mid-buccal aspect from the cemento-enamel junction to the bottom of the gingival recession using a PCP 12 periodontal probe. Thereafter, reference notches were prepared on the buccal aspect of respective root surfaces by the use of a small diamond burr to mark the coronal extension of the gingival margin (GM) at baseline. This landmark served as reference point for the histomorphometrical analysis (Fig. 1a).

The coverage of gingival recessions was performed according to a modification of a standard technique [12]. In brief, intrasulcular incisions were performed at $\mathrm{C}$ as well as the 
Fig. 1 a Reference notches were prepared using a small diamond burr to mark the coronal extension of the gingival margin at chronic recession-type defects (Miller

Class I-like). b CTG harvested from the palate was adapted to the partial-thickness gingival flap by a single resorbable mattress suture. c CM was applied in a way identical to CTG but with its spongious part facing the periosteum. d The gingival flaps were advanced in coronal direction exceeding the notch area by $2-3 \mathrm{~mm}$. e Mattress and sling sutures secured the coronally advanced flaps. f Clinical situation at 12 weeks $(\mathrm{CAF}+\mathrm{CM}$, refers to a) indicating that the oral hygiene program was associated with low plaque scores. Complete coverage of the notch area was achieved

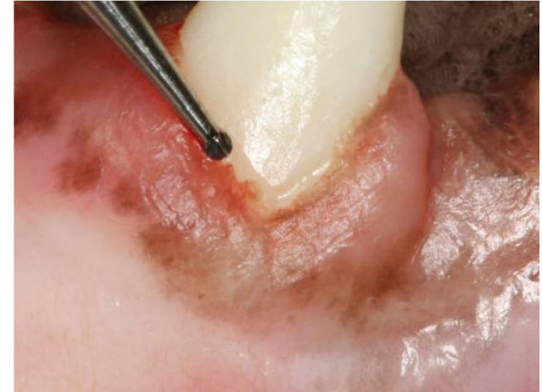

$\mathbf{a}$
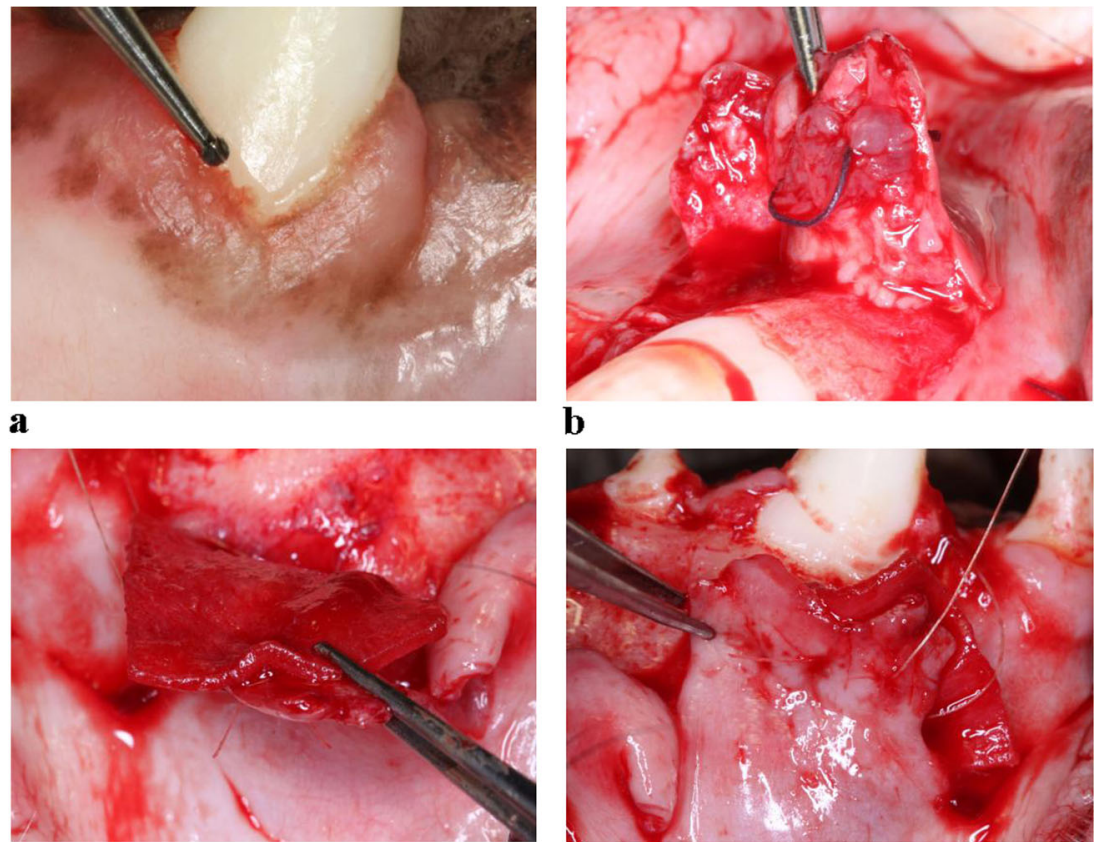

c

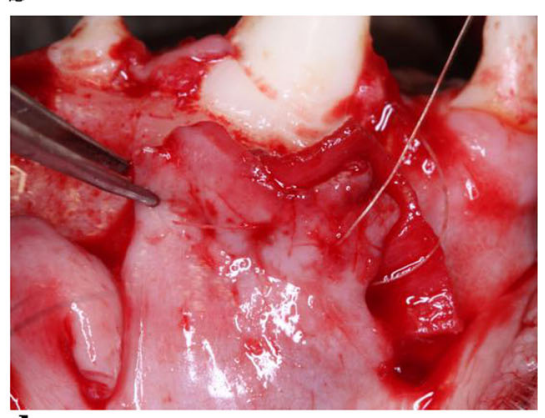

d

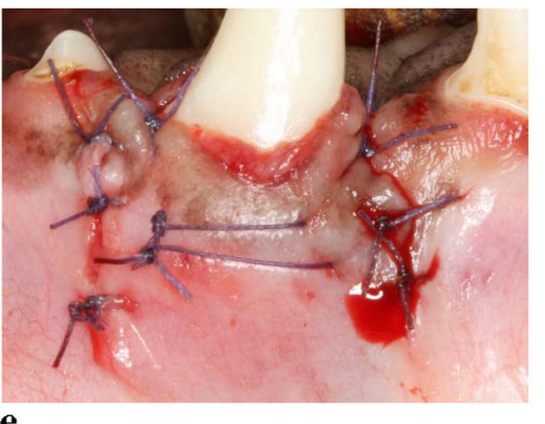

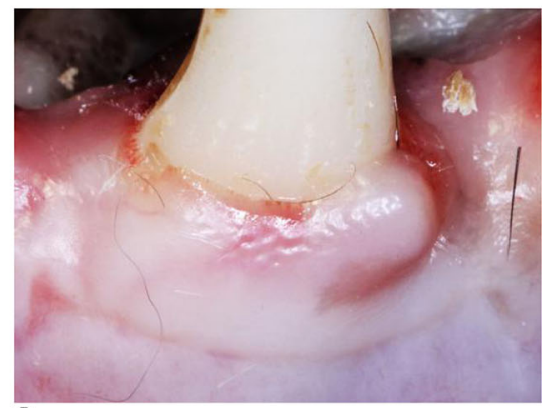

f mesial roots of $\mathrm{P} 3$ and $\mathrm{P} 4$. This was followed by the elevation of a partial-thickness gingival flap beyond the mucogingival junction employing two vertical releasing incisions. The facial portions of the adjacent papillae were de-epithelialized by the use of a fine-grit diamond burr to allow a coronal repositioning of the flap. At respective sites, a porcine-derived, native CM (Mucograft ${ }^{\circledR}$; Geistlich Biomaterials, Wolhusen, Switzerland) was trimmed and adapted over the entire dehiscence area so as to cover $1-2 \mathrm{~mm}$ of the surrounding connective tissue bed at the recipient site. Free CTGs (size and thickness corresponding to $\mathrm{CM}$ ) were bilaterally harvested from the palate in the region of P3 to M2 according to the technique described by Vignoletti et al. [11] and applied in a way identical to CM. Both CM (i.e., compact layer) and CTG were sutured to the mucosal flap (Resorba ${ }^{\circledR}$; Nürnberg, Germany) (Fig. 1b and c). Finally, at all sites, the gingival flaps were advanced, repositioned about $2-3 \mathrm{~mm}$ coronal to $\mathrm{N}$ and fixed with vertical/horizontal mattress as well as sling sutures $\left(\right.$ Resorba $\left.^{\circledR}\right)$ (Fig. 1d and e). At 7 days after surgery, tooth and implant cleaning was initiated and maintained by the use of a toothbrush three times per week (Fig. 1f). All experimental procedures were performed by two experienced surgeons (F.S. and I.M.).

\section{Retrieval of specimens}

The animals were euthanized (overdose of sodium pentobarbital $3 \%$ ) at 12 weeks after the final surgical intervention. The oral tissues were fixed by perfusion with $10 \%$ buffered formalin administered through the carotid arteries. The jaws were dissected and blocks containing the experimental specimens were obtained. All specimens were fixed in $10 \%$ neutral buffered formalin solution for 4-7 days.

\section{Histological preparation}

The block biopsies containing the experimental teeth were decalcified in ethylenediaminetetraacetic acid under radiographic control of the decalcification process, dehydrated, and fixed in paraffin. Vestibulo-oral serial sections were cut parallel to the long-axis of the teeth with the micrometer set at $5 \mu \mathrm{m}$. Sections representing the central parts of each root were 
Table 1 Mean values $( \pm \mathrm{SD})$ of $\mathrm{CDH}, \mathrm{N}-\mathrm{GM}$, GM-aJE, aJE-BC, and GT (in $\mathrm{mm} \pm \mathrm{SD}$ ) after 12 weeks in different groups at buccal aspects: upper jaw $(n=6$ dogs; $\mathrm{CAF}, n=6 ; \mathrm{CAF}+\mathrm{CTG}, n=6 ; \mathrm{CAF}+\mathrm{CM}, n=6)$

\begin{tabular}{llllrl}
\hline Group & CDH & N-GM & GM-aJE & aJE-BC & GT \\
\hline CAF+CM & $1.54 \pm 0.81$ & $1.29 \pm 0.66$ & $2.48 \pm 0.68$ & $1.08 \pm 0.15$ & $0.79 \pm 0.30$ \\
CAF+CTG & $1.66 \pm 0.33$ & $0.76 \pm 1.18$ & $2.23 \pm 0.93$ & $1.21 \pm 0.37$ & $0.64 \pm 0.40$ \\
CAF & $1.14 \pm 0.71$ & $1.68 \pm 0.31$ & $1.97 \pm 0.68$ & $1.03 \pm 0.25$ & $0.94 \pm 0.20$ \\
\hline
\end{tabular}

$P>0.05$; ANOVA, respectively

selected for the histological analysis in each group. Finally, the sections were stained with toluidine blue and basic fuchsin.

Histological analysis

The most central sections of each tooth were chosen for morphologic and morphometric evaluation. For image acquisition a ProgRes ${ }^{\circledR}$ C5 digital camera (JENOPTIK Optical Systems GmbH, Jena, Germany) connected to a Zeiss Axioplan microscope (Carl Zeiss, Göttingen, Germany) was used. Digital images were evaluated using a software program (ProgRes ${ }^{\circledR}$ Mac CapturePro 2.7; JENOPTIK Optical Systems GmbH, Jena, Germany).

The following landmarks were identified in the stained sections at the buccal aspect: apical border of the notch area $(\mathrm{N})$, gingival margin (GM), the apical extension of the long junctional epithelium (aJE), and bone crest (BC). Linear measurements were made by drawing a vertical line, following the long axis of the tooth, from $\mathrm{N}$ to GM, GM to aJE, and aJE to BC. In addition, gingival thickness (GT) was measured perpendicular to the root surface by drawing a horizontal line from $\mathrm{N}$ to the outer contour of the adjacent soft tissue.

All histological measurements were performed by one experienced and calibrated investigator (Y.S.) masked to the specific experimental conditions.

Statistical analysis

The statistical analysis was performed using a commercially available software program (PASW Statistics 20.0; SPSS Inc., Chicago, IL, USA). Mean values and standard deviations among animals were calculated for each variable and group. The data rows were examined with the Kolmogorow-
Smirnow test for normal distribution. One-way analysis of variance (ANOVA) and post-hoc testing with Bonferroni's correction for multiple comparisons was used for between group comparisons. The alpha error was set at 0.05 .

\section{Results}

At the end of the chronification period, a total of three experimental sites (all lower jaws) revealed a spontaneous regeneration of the acute gingival dehiscence-type defects. These sites were excluded from the experimental procedures. Thus, a total of 33 defects created in the upper and lower jaws of six animals were randomly allocated to three treatment procedures (upper jaw: $\mathrm{CAF}=6 ; \mathrm{CAF}+\mathrm{CTG}=6 ; \mathrm{CAF}+\mathrm{CM}=6$; lower jaw: $\mathrm{CAF}=6 ; \mathrm{CAF}+\mathrm{CTG}=3 ; \mathrm{CAF}+\mathrm{CM}=6)$. Mean baseline $\mathrm{CDH}$ values in both upper and lower jaws were comparable in all groups investigated $(P>0.05$; ANOVA, respectively) (Tables 1, 2 and 3).

The postoperative healing was uneventful in all animals. No complications such as allergic reactions, abscesses or infections were noted throughout the entire study period. The experimental procedures had no influence on the health status, behaviour or feeding habit of each animal. The oral hygiene program successfully prevented plaque biofilm formation at the experimental sites.

Histomorphometrical analysis/histological observations

After 12 weeks of healing, both clinical and histological analysis has indicated that the CAF procedure was, in most of the specimens, associated with a partial or complete soft

Table 2 Mean values $( \pm \mathrm{SD})$ of CDH, N-GM, GM-aJE, aJE-BC, and GT (in $\mathrm{mm} \pm \mathrm{SD}$ ) after 12 weeks in different groups at buccal aspects: lower jaw $(n=6$ dogs; $\mathrm{CAF}, n=6 ; \mathrm{CAF}+\mathrm{CTG}, n=3 ; \mathrm{CAF}+\mathrm{CM}, n=6)$

\begin{tabular}{|c|c|c|c|c|c|}
\hline Group & $\mathrm{CDH}$ & N-GM & GM-aJE & aJE-BC & GT \\
\hline $\mathrm{CAF}+\mathrm{CM}$ & $1.58 \pm 0.17$ & $0.79 \pm 0.68^{\mathrm{a}}$ & $2.09 \pm 0.53$ & $1.05 \pm 0.25$ & $0.57 \pm 0.35$ \\
\hline $\mathrm{CAF}+\mathrm{CTG}$ & $1.33 \pm 0.67$ & $1.95 \pm 0.34$ & $2.07 \pm 0.33$ & $0.89 \pm 0.08$ & $1.01 \pm 1.43$ \\
\hline CAF & $1.46 \pm 0.87$ & $1.18 \pm 0.45$ & $2.05 \pm 0.33$ & $0.89 \pm 0.17$ & $0.64 \pm 0.18$ \\
\hline
\end{tabular}

${ }^{\mathrm{a}} \mathrm{CAF}+\mathrm{CM}$ vs. $\mathrm{CAF}+\mathrm{CTG}, P<0.05$; ANOVA 
Table 3 Mean values ( \pm SD) of CDH, N-GM, GM-aJE, aJE-BC, and GT (in $\mathrm{mm} \pm \mathrm{SD}$ ) after 12 weeks in different groups at buccal aspects: upper and lower jaws ( $n=6$ dogs; $\mathrm{CAF}, n=12 ; \mathrm{CAF}+\mathrm{CTG}, n=9 ; \mathrm{CAF}+\mathrm{CM}, n=12)$

\begin{tabular}{llllll}
\hline Group & CDH & N-GM & GM-aJE & aJE-BC & GT \\
\hline CAF+CM & $1.55 \pm 0.65$ & $1.04 \pm 0.69$ & $2.28 \pm 0.62$ & $1.06 \pm 0.20$ & $0.68 \pm 0.33$ \\
CAF+CTG & $1.51 \pm 0.50$ & $1.15 \pm 1.12$ & $2.18 \pm 0.76$ & $1.10 \pm 0.34$ & $0.76 \pm 0.37$ \\
CAF & $1.27 \pm 0.76$ & $1.43 \pm 0.45$ & $2.01 \pm 0.51$ & $0.96 \pm 0.22$ & $0.79 \pm 0.24$ \\
\hline
\end{tabular}

$P>0.05$; ANOVA, respectively

tissue coverage on a level above the reference point (i.e., $\mathrm{N}$ ) (Figs. 1f and 2).

Only at two sites, GM was either located on a level equivalent to $(n=1$, upper jaw, $\mathrm{CAF}+\mathrm{CM})$ or even below $\mathrm{N}(n=1$, lower jaw, $\mathrm{CAF}+\mathrm{CM}$ ), thus pointing to a certain retraction of GM during the healing period of 12 weeks. Histological analysis failed to identify any residues of CM or CTG as well as any new cementum formation in the notch area of all specimens investigated (Fig. 3).

The histomorphometrical parameters assessed at the buccal aspects of experimental teeth in both upper and lower jaws are summarized in Tables 1, 2, and 3.

While between group comparisons of mean N-GM, GMaJE, aJE-BC and GT values revealed no significant differences in the upper jaw, statistical analysis of the lower jaw has pointed to significant differences in mean N-GN and GT values. In particular, mean $\mathrm{N}-\mathrm{GM}$ values were significantly higher in the $\mathrm{CAF}+\mathrm{CTG}$ when compared either with $\mathrm{CAF}+$ $\mathrm{CM}$ or CAF groups $(P<0.05$; unpaired $t$-test, respectively). Similarly, mean GT values were significantly higher when comparing $\mathrm{CAF}+\mathrm{CTG}$ with $\mathrm{CAF}$ alone $(P<0.05$; unpaired $t$-test) (Tables 1 and 2). However, the statistical analysis of

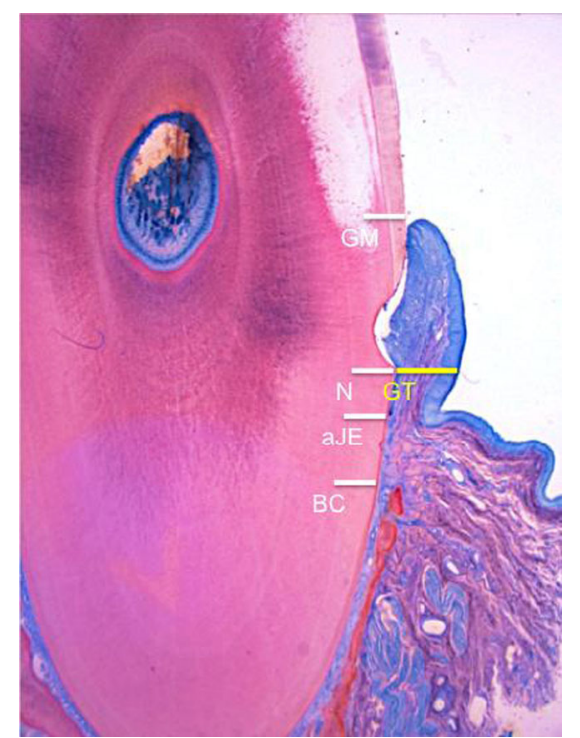

Fig. 2 The following landmarks were identified in the stained sections: $a J E$ the apical extension of the long junctional epithelium, $B C$ bone crest, $G M$ gingival margin, $N$ apical border of the notch area (CAF treated site, upper jaw, original magnification $\times 12$ ) mean N-GM, GM-aJE, aJE-BC and GT values calculated from both upper and lower jaws failed to identify any significant differences between all surgical procedures investigated (Table 3).

Between group comparisons in the upper jaw failed to reveal any significant differences in mean N-GM, GM-aJE, aJE-BC or GT values (Table 1). In the lower jaw, statistical analysis has pointed to significantly higher mean N-GM values in the $\mathrm{CAF}+\mathrm{CTG}$ group when compared with $\mathrm{CAF}+$ $\mathrm{CM}$ treated sites $(P<0.05$; ANOVA). Even though mean GT values tended to be highest in the $\mathrm{CAF}+\mathrm{CTG}$ group, these differences failed to reach statistical significance over either $\mathrm{CAF}$ or $\mathrm{CAF}+\mathrm{CM}$ treated sites $(P>0.05$; ANOVA, respectively) (Table 2). Similarly, the statistical analysis of mean NGM, GM-aJE, aJE-BC and GT values calculated from both upper and lower jaws failed to identify any significant differences between all surgical procedures investigated $(P>0.05$; ANOVA, respectively) (Table 3).

\section{Discussion}

The present study was designed to evaluate histologically the healing following treatment of localized gingival recessions using CAF either alone or combined with CM or CTG in a canine model [13]. Basically, after 12 weeks of healing, it was observed that all three reconstructive procedures resulted in comparable recession coverage, as identified by mean N-GM values. This was particularly true for the defects localized in upper jaws. When considering the outcomes in lower jaws, mean $\mathrm{N}-\mathrm{GM}$ values were highest at $\mathrm{CAF}+\mathrm{CTG}$ treated sites, even reaching statistical significance over $\mathrm{CAF}+\mathrm{CM}$ and CAF groups, respectively. However, the vast majority of sites was associated with complete a complete coverage of the $\mathrm{N}$ area in both upper (CAF: $100 \%$; $\mathrm{CAF}+\mathrm{CM}: 83.3 \%$; $\mathrm{CAF}+$ CTG: $100 \%$ ) and lower (CAF: $100 \%$; $\mathrm{CAF}+\mathrm{CM}: 83.3 \%$; $\mathrm{CAF}+\mathrm{CTG}$ : $100 \%$ ) jaws, and this outcome may be of a higher clinical relevance than mean N-GM values alone.

However, when interpreting these outcomes, it hast to be kept in mind out that three defects in the lower jaw revealed a spontaneous healing during the chronification period, thus pointing to the high healing potential of this types of defects. 


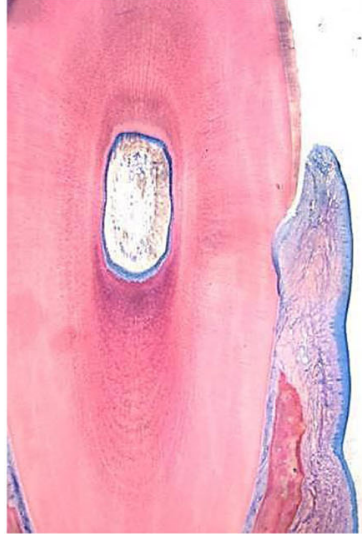

a

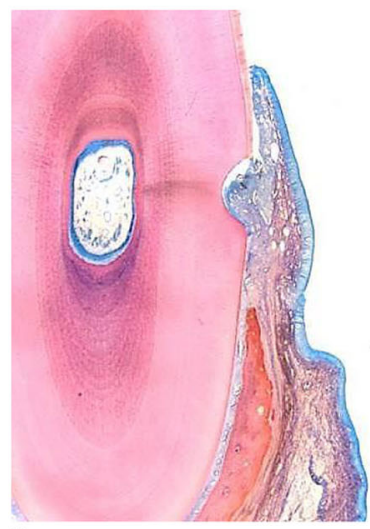

c

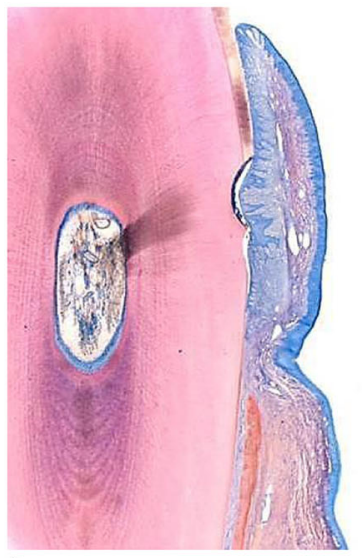

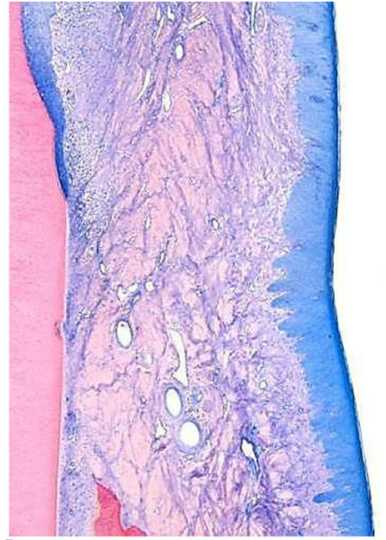

b

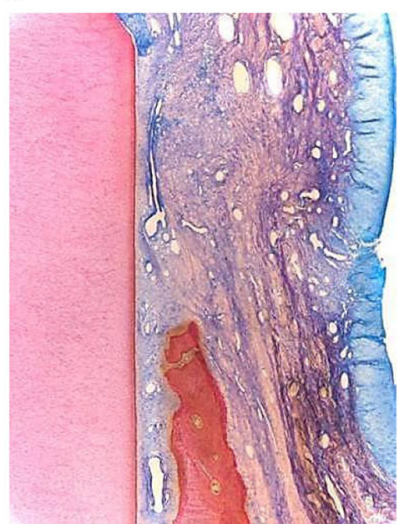

d

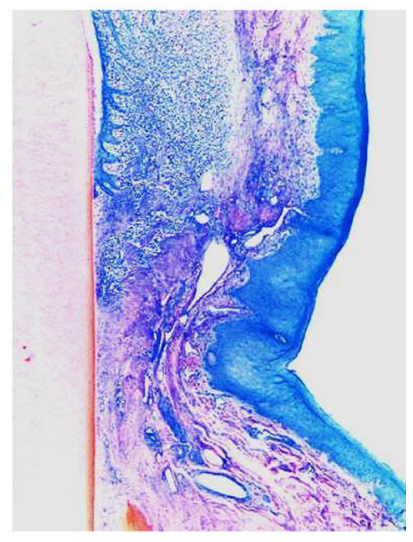

f
Fig. 3 Representative histological views of wound healing in different groups at 12 weeks. All treatment procedures investigated were associated with an almost complete soft tissue coverage of the defect area. a $\mathrm{CAF}+\mathrm{CM}$ (lower jaw, original magnification $\times 12$ ). b Higher magnification $\times 25$ view of the buccal aspect shown in panel a (specimen refers to experimental site shown in Fig. 1f). c CAF+CTG (lower jaw, original magnification $\times 12$ ). d Higher magnification $\times 25$ view of the buccal aspect shown in panel c. e $\mathrm{CAF}+\mathrm{CM}$ (lower jaw, original magnification $\times$

12). $\mathbf{f}$ Higher-magnification $(\times 25)$ view of the buccal aspect shown in panel e. Residues of CM were commonly not observed

Therefore, future studies employing this animal model should rather consider the creation of more extensive defects such as Miller Class II recessions which may mimic closer the clinical situation.
The observation that both $\mathrm{CAF}+\mathrm{CM}$ and $\mathrm{CAF}$ alone may result in comparable outcomes in terms of root coverage is also supported by previous findings from a preclinical study performed in minipigs [11]. Moreover, the observation that $\mathrm{CAF}, \mathrm{CAF}+\mathrm{CM}$, and $\mathrm{CAF}+\mathrm{CTG}$ may result in a substantial coverage of localized recession defects is in agreement with the results of recent clinical studies $[6,9,10]$. McGuire and Scheyer [9] reported that at 6 months after therapy, the mean change in recession depth from baseline amounted to $2.62 \mathrm{~mm}$ in the $\mathrm{CAF}+\mathrm{CM}$ group, and $3.10 \mathrm{~mm}$ in the $\mathrm{CAF}+\mathrm{CTG}$ group, the difference between the groups being statistically significant. At 12 months, the percentage of root coverage amounted to $88.5 \%$ in the $\mathrm{CAF}+\mathrm{CM}$ group, and $99.3 \%$ at $\mathrm{CAF}+\mathrm{CTG}$ treated sites, the difference being again statistically significant [9]. On the other hand, keratinized tissue width gains were equivalent for both therapies and averaged $1.34 \mathrm{~mm}$ for $\mathrm{CAF}+\mathrm{CM}$ treated sites and $1.26 \mathrm{~mm}$ in the $\mathrm{CAF}+\mathrm{CTG}$ group. No statistically significant differences between subject-reported values for esthetic satisfaction, and subjects' assessments of pain and discomfort were found between the two treatments. Comparable outcomes were also reported by Cardaropoli et al. [10]. However, in that study percentage of root coverage amounted to $94.32 \%$ in the $\mathrm{CAF}+\mathrm{CM}$ group and $96.97 \%$ in the $\mathrm{CAF}+\mathrm{CTG}$ group, respectively, without any statistically significant difference in any clinical parameter.

In a recent multicenter study, at 6 months after therapy, root coverage amounted to $75.29 \%$ in the $\mathrm{CAF}+\mathrm{CM}$ group and slightly, but not statistically significant, lower at sites undergoing CAF alone (72.66 \%). The application of CM appeared to be more beneficial in recessions larger than $3 \mathrm{~mm}(72.03 \%$ vs. $66.16 \%)[6]$.

In the present study, all three treatment procedures resulted in a comparable healing pattern as indicated by the histological analysis (e.g., no statistically significant differences in mean GM-aJE and aJE-BC values and complete lack of cementum formation). These observations are somewhat in contrast with previous findings in minipigs where after a healing period of 12 weeks, treatment with $\mathrm{CAF}+\mathrm{CM}$ was associated with a shorter dimension of the junctional epithelium $(2.26 \pm 0.23$ vs. $2.79 \pm 0.77 \mathrm{~mm})$ and higher amount of cementum formation in the former defect area $(1.08 \pm 0.41$ vs. $0.75 \pm 0.25 \mathrm{~mm})$ when compared with CAF alone [11]. However, it must be realized that the present study had as primary focus the evaluation of soft tissue (i.e., gingival) healing and not of periodontal wound healing/regeneration. For this reason, the reference notches prepared on the buccal aspect of experimental root surfaces were meant to indicate the level of the GM and not the most apical level of the exposed root surfaces and thus, the defect area demarcated by the notch (i.e., N) is not primarily suitable to evaluate formation of cementum and periodontal ligament. 
The soft tissue thickness, as histomorphometrically estimated by mean GT values, was heterogeneously distributed, but tended to be highest at CAF treated sites in the upper-, and $\mathrm{CAF}+\mathrm{CTG}$ treated sites in the lower jaws. The observation that neither CM nor CTG resulted in an improvement of the overall (i.e., upper and lower jaws) GT values is, to a certain extend, in disagreement with previous preclinical and clinical observations [6, 13]. In particular, soft tissue thickness after coverage of mucosal recessions in the upper jaw tended to be higher in the $\mathrm{CAF}+\mathrm{CM}(0.71 \pm 0.55 \mathrm{~mm})$ and $\mathrm{CAF}+\mathrm{CTG}$ $(0.71 \pm 0.55 \mathrm{~mm})$ groups, when compared with $\mathrm{CAF}$ alone $(0.34 \pm 0.23 \mathrm{~mm})$ [13]. Similarly, in a multicenter study, $\mathrm{CAF}+\mathrm{CM}$ was even associated with significantly more gain in GT than CAF (0.59 vs. $0.34 \mathrm{~mm}$ ) [6]. The discrepancy noted between these studies may primarily be explained by potential differences in the initial intra- and interspecies soft tissue thickness. Moreover, one has to realize that histological and clinical assessments may not be reliable to estimate the exact change in soft tissue thickness, and therefore, future studies should consider different methodologies and technologies, such as 3D digital imaging analysis [14].

Taken together, the present results indicate that in the used defect model, the application of CTG or CM in conjunction with CAF may not have an advantage over the use of CAF alone thus suggesting that $\mathrm{CAF}$ alone is still a valuable option for the treatment isolated Miller Class I gingival recessions.

Acknowledgments We kindly appreciate the skills and commitment of Mr. Vladimir Golubovic and Mr. Thomas Kaiser (Department of Oral Surgery, Heinrich Heine University) in the preparation of the histological specimens. The study was funded by an unrestricted grant of the Osteology Foundation, Lucerne, Switzerland.

Conflict of interest The authors declare that they have no conflict of interests related to this study.

\section{References}

1. Nieri M, Pini Prato GP, Giani M, Magnani N, Pagliaro U, Rotundo R (2013) Patient perceptions of buccal gingival recessions and requests for treatment. J Clin Periodontol 40:707-712

2. Cairo F, Pagliaro U, Nieri M (2008) Treatment of gingival recession with coronally advanced flap procedures: a systematic review. J Clin Periodontol 35:136-162
3. Roccuzzo M, Bunino M, Needleman I, Sanz M (2002) Periodontal plastic surgery for treatment of localized gingival recessions: a systematic review. J Clin Periodontol 29(Suppl 3):178-194

4. Griffin TJ, Cheung WS, Zavras AI, Damoulis PD (2006) Postoperative complications following gingival augmentation procedures. J Periodontol 77:2070-2079

5. Schwarz F, John G, Kaiser T, Mihatovic I, Golubovic V, Becker J (2013) Impact of proangiogenic factors on organization and biodegradation of a collagen matrix. An immunohistochemical study in rats. Clin Oral Implants Res. doi:10.1111/clr.12211

6. Jepsen K, Jepsen S, Zucchelli G, Stefanini M, de Sanctis M, Baldini N, Greven B, Heinz B, Wennstrom J, Cassel B, Vignoletti F, Sanz M (2013) Treatment of gingival recession defects with a coronally advanced flap and a xenogeneic collagen matrix: a multicenter randomized clinical trial. J Clin Periodontol 40:82-89

7. Aroca S, Molnar B, Windisch P, Gera I, Salvi GE, Nikolidakis D, Sculean A (2013) Treatment of multiple adjacent Miller class I and II gingival recessions with a Modified Coronally Advanced Tunnel (MCAT) technique and a collagen matrix or palatal connective tissue graft: a randomized, controlled clinical trial. J Clin Periodontol 40: 713-720

8. Molnar B, Aroca S, Keglevich T, Gera I, Windisch P, Stavropoulos A, Sculean A (2013) Treatment of multiple adjacent Miller Class I and II gingival recessions with collagen matrix and the modified coronally advanced tunnel technique. Quintessence Int 44:17-24

9. McGuire MK, Scheyer ET (2010) Xenogeneic collagen matrix with coronally advanced flap compared to connective tissue with coronally advanced flap for the treatment of dehiscence-type recession defects. J Periodontol 81:1108-1117

10. Cardaropoli D, Tamagnone L, Roffredo A, Gaveglio L (2012) Treatment of gingival recession defects using coronally advanced flap with a porcine collagen matrix compared to coronally advanced flap with connective tissue graft: a randomized controlled clinical trial. J Periodontol 83:321-328

11. Vignoletti F, Nunez J, Discepoli N, De Sanctis F, Caffesse R, Munoz F, Lopez M, Sanz M (2011) Clinical and histological healing of a new collagen matrix in combination with the coronally advanced flap for the treatment of Miller class-I recession defects: an experimental study in the minipig. J Clin Periodontol 38:847-855

12. Allen EP, Miller PD Jr (1989) Coronal positioning of existing gingiva: short term results in the treatment of shallow marginal tissue recession. J Periodontol 60:316-319

13. Schwarz F, Mihatovic I, Shirakata Y, Becker J, Bosshardt D, Sculean A (2014) Treatment of soft tissue recessions at titanium implants using a resorbable collagen matrix: a pilot study. Clin Oral Implants Res 25:110-115

14. Thoma DS, Jung RE, Schneider D, Cochran DL, Ender A, Jones AA, Görlach C, Uebersax L, Graf-Hausner U, Hämmerle CH (2010) Soft tissue volume augmentation by the use of collagen-based matrices: a volumetric analysis. J Clin Periodontol 37:659-666 